\title{
DOS COPISTAS EN EL SIGLO X: GÓMEZ DE CARDEÑA Y GÓMEZ DE ALBELDA
}

\author{
POR \\ PALOMA CUENCA MUÑOZ ${ }^{1}$ \\ Universidad Complutense de Madrid
}

\begin{abstract}
RESUMEN
Durante la primera mitad del siglo X se realiza en el monasterio de San Pedro de Cardeña un códice que contiene los Moralia in lob de san Gregorio y en San Martín del Albelda se copia la obra de san Ildefonso De virginitate perpetua. Ambos manuscritos están realizado por un copista llamado Gómez. La crítica ha planteado en algunos trabajos la posibilidad de que ambos copistas fueran el mismo, sin embargo, el presente trabajo expone mediante un análisis paleográfico la mayor probabilidad de que se trate de dos copistas diferentes.
\end{abstract}

PALABRAS CLAVE: paleografía; Alta Edad Media; escritura visigótica; códices medievales; codicología; san Gregorio; san Ildefonso.

\section{TWO COPYISTS IN THE TENTH CENTURY: GÓMEZ FROM CARDEÑA AND GÓMEZ FROM ALBELDA}

\begin{abstract}
During the first half of the Xth century a codex which contains the Moralia in lob written by saint Gregory, was performed in the monastery of san Pedro de Cardeña. Another codex, De virginitate perpetua by san Ildefonso, was copied by a scribe in the monastery of san Martín de Albelda, also during the first half. Both manuscripts were performed by a copyist called Gómez. The review has raised in some works the possibility that both copyists were de the same however through a paleographic analysis this research proposes a greater probability that the copyists are different.
\end{abstract}

KEY WORDS: Paleography; High Middle Ages; Visighotic writing; Medieval codices; Codicology; Saint Gregory; Saint Ildefonso.

Cómo CITAR eSTE ARTículo / CITATION: Cuenca Muñoz, P. 2018. «Dos copistas en el siglo X: Gómez de Cardeña y Gómez de Albelda». Hispania Sacra 70, 142: 423-431. https://doi.org/10.3989/hs.2018.028

Recibido/Received 28-08-2017

Aceptado/Accepted 22-05-2018

\section{INTRODUCCIÓN}

Según propone el profesor José Antonio Fernández Flórez «puede [un códice], finalmente, haber surgido como consecuencia de una cierta actividad comercial, desencadenada a partir de las peticiones de otra personas individuales o colectivas, que, a su vez, habrían solicitado la copia de una obra específica». ${ }^{2}$ Su propuesta describe exactamente la génesis del ms. lat. 2855, conservado en la actualidad en la Bibliothèque Nationale de France. Cuando el obispo Gotescalco viaja ente los años 950 a 951 desde

1 palomacm@ucm.es / ORCID iD: http://orcid.org/0000-00033776-9940

2 Fernández Flórez 1977: 159-160.
Aquitania a Santiago de Compostela y vuelve a su ciudad de origen, realiza una parada en el monasterio de san Martín de Albelda, dónde le encarga a un escriba llamado Gómez que realice una copia de la obra de san Ildefonso De virginitate perpetua sanctae Mariae. ${ }^{3}$ Unos años antes, en el 914, y a solo cierta distancia de allí, en el monasterio de san Pedro de Cardeña, un monje también llamado Gómez, terminó de copiar un ejemplar de los Moralia in lob de

3 Son numerosos los trabajos que han estudiado este códice desde diferentes ámbitos, en el presente estudio se han tenido en cuenta desde los más antiguos de Cantera Orive 1948-1949: 299-304 y 427-442; hasta el más reciente de Yarza Urquiola 2016: 213-232; sin olvidar las valiosas aportaciones de los profesores M. C. Díaz y Díaz, J. A. Fernández Flórez y M. Herrero de la Fuente, entre otros. 
san Gregorio Magno, en este caso parece que para un uso interno colectivo del propio centro.

El presente trabajo nace para intentar aportar nuevos argumentos a la luz de los estudios actuales sobre un problema que abordaron dos grandes investigadores: Justo Pérez de Urbel, quien se inclina a pensar que Gómez es el mismo copista en san Pedro de Cardeña y en san Martín de Albelda, y Manuel C. Díaz y Díaz, que se resiste a aceptar dicha afirmación, más por intuición que por un análisis de la escritura. A partir de la homonimia y de las cercanas coordenadas espacio-temporales, es posible plantearse si existen argumentos para afirmar que ambos códices están realizados por la misma mano, o si por el contario, existen datos que hagan pensar en una simple coincidencia. ${ }^{4}$

La suposición de que el escriba albeldense Gómez, quien añade un prólogo al texto de san Ildefonso y a continuación escribe la obra, sea la misma persona que Gómez, diaconus probablemente de Cardeña que copia los Moralia, viene planteada a partir de las relaciones que la historiografía ha establecido entre ambos cenobios durante la primera mitad del siglo X. Así, se parte de considerar a san Pedro de Cardeña la abadía madre de san Martín de Albelda, llegando incluso a afirmar que Pedro, abad de Cardeña, en el año 921 fue nombrado también el primer abad de Albelda como superioridad de Gabelo, y por lo tanto, llegó a ser abad de los dos monasterios a la vez. ${ }^{5}$ En este mismo sentido se refiere Justo Pérez de Urbel al «préstamo de personal» de Cardeña hacia Albelda, llegando a mencionar a un personaje llamado Gómez, aunque sin aclarar si se refiere al escriba o al abad del mismo nombre. Ahora bien, la referencia a la posibilidad que aborda el presente trabajo queda muy clara en el siguiente párrafo:

En tiempo de Dulquito, Albelda tenía ya cerca de doscientos monjes, lo mismo que San Pedro de Cardeña. Entre ellos estaba el copista Gómez, que nos recuerda a Gómez de Cardeña, si no es el mismo Gómez diácono y peccador ( $\mathrm{sic}$ ) que treinta años antes había terminado de escribir los Morales del Papa San Gregorio. Y si así fuese, podríamos considerarle como el enlace cultural entre el condado de Castilla, que empezaba a organizarse, y la Rioja, recientemente conquistada. Aun conservamos el códice que en 951 dio al obispo Godescalco de Puy, a su paso para Compostela, con el libro de San Ildefonso la Virginidad perpetua de la Santísima Virgen. Él fue, sin duda, el que juntamente con Salvo, nombrado abad del monasterio dos años más tarde, formó y organizó el escritorio famoso del que había de salir uno de los manuscritos más brillantes de aquel tiempo. ${ }^{6}$

\footnotetext{
4 Pérez de Urbel 1977: 89; Díaz y Díaz 1979: 62, n. 28.

5 Esta afirmación se debe a uno de los investigadores que más ha difundido la dependencia entre ambos monasterios, Pérez de Urbel 1950: 495-534. Más recientemente ha trabajado sobre la historia de este monasterio García Turza 2002: 9-27.

6 Pérez de Urbel 1950: 519; además en la nota número 2, en esta misma página, insiste: «El Gómez "diaconus, peccator" que en 914 escribió los Morales de San Gregorio, podría muy bien ser el Gómez presbítero que en 950 tenía terminado De virginitate Beatae Mariae». En el mismo sentido De Silva y Verástegui 1984: 44, al analizar el influjo del entrelazado nórdico en el códice de Albelda propone «que fue precisamente a través de Castilla, como estas formas carolingias llegaron a aquélla. Aunque no sabemos nada sobre la personalidad del escriba Gómez, admitiendo que él mismo realizara la decoración del códice y la posibilidad de que fuera el mismo escriba que en el año 914 terminó los
}

Como se expone en el pasaje citado de Pérez de Urbel, los dos códices podrían, por tanto, ser obra de la misma mano, sin embargo el análisis paleográfico de ambos textos demuestra que la coincidencia en la identidad de ambos copistas sería bastante improbable.

\section{LOS DOS CÓDICES}

Si establecemos un primera comparativa desde el ámbito codicológico, se observa en el siguiente cuadro las numerosas diferencias que se pueden establecer entre el códice de los Moralia, ms. lat. 83, conservado en la John Rylands Library de Manchester y el ms. lat. 2855 de la Biliothèque Nationale de France. ${ }^{7}$ No se tienen en cuenta los datos que aportan las encuadernaciones de dicho códices, ya que en ambos casos nada tienen que ver con el manuscrito visigótico original. Así, la del manuscrito de san Gregorio fue destruida junto con el principio y el final del texto, y el ejemplar de san Ildefonso forma parte de un códice facticio del que es una pieza independiente que ocupa tan solo los folios 69 a 160.

\begin{tabular}{|c|c|}
\hline Ms. Lat. 83 (J. R. L.) & Ms. Lat. 2855 (B. N. F) \\
\hline Medidas $\quad 415-420 \times 310 \mathrm{~mm}$. & $205^{\times} 145 \mathrm{~mm}$ \\
\hline $\begin{array}{ll}\text { Disposición } & \text { dos columnas } \\
\text { Marginalia en visigótica } & \text { con llamadas }\end{array}$ & $\begin{array}{l}\text { a línea tirada } \\
\text { sin llamadas }\end{array}$ \\
\hline $\begin{array}{r}1 \text { bifolio } \\
\text { (parte de un cuaternión) } \\
41 \text { cuaterniones } \\
1 \text { senio }\end{array}$ & $\begin{array}{r}1 \text { binión } \\
11 \text { cuaterniones (escritura en e } \\
\text { último vuelto y en el folio de } \\
\text { guarda }\end{array}$ \\
\hline $\begin{array}{l}\text { Numeración Cuadernos numerados con } \\
\text { numerales romanos }\end{array}$ & $\begin{array}{r}\text { Cuadernos sin numerar, divididos } \\
\text { por reclamos }\end{array}$ \\
\hline $\begin{array}{l}\text { Texto añadido en escritura visigótica con } \\
\text { marcas de omisión }\end{array}$ & $\begin{array}{l}\text { Texto añadido en escritura } \\
\text { visigótica sin marcas de omisión }\end{array}$ \\
\hline Material escriptorio Pergamino grueso & Pergamino finc \\
\hline $\begin{array}{lr}\text { Estado de conservación } & \text { Irregular } \\
\text { Tratamiento/calidad } & \text { Bueno }\end{array}$ & $\begin{array}{l}\text { Excelente } \\
\text { Excelente }\end{array}$ \\
\hline
\end{tabular}

Según se muestra en el cuadro anterior, el aspecto físico de ambos códices presenta suficientes características diferenciadoras entre sí, como para no establecer ningún tipo de relación entre ellos desde el ámbito puramente material, más allá del hecho de tratarse de dos códices escritos en zonas peninsulares cercanas en el siglo X.

Por lo que se refiere a la forma interna de concebir $y$ estructurar la copia de la obra, tampoco se encuentran concomitancias entre la obra de san Ildefonso y la copia gregoriana. Si bien es cierto que ambos textos incluyen en su inicio algunas obras menores a modo de prólogo, mientras que el De virginitate va precedido de una breve plegaria, obra quizás de un clérigo llamado Abraam, del famoso

Moralia de San Gregorio en el monasterio castellano de San Pedro de Cardeña, tendríamos en él el enlace cultural entre Castilla y Albelda».

7 Los datos codicológicos que se utilizan en este estudio provienen en el caso del ms. lat. 83, Moralia in lob de san Gregorio, del artículo de Cuenca Muñoz 2018: 167-195. Por su parte, los del ms. lat. 2855, De virginitate perpetua sanctae Mariae de san Ildefonso, remiten a la edición del texto Blanco García 1971; así como al excelente estudio de Díaz y Díaz 2002: 49-76. 
prólogo que firma el propio Gómez, además de dedicar unos folios a copiar el Elogium Ildefonsi que realizara Julián; el manuscrito copiado unos años antes que conforma el códice de los Moralia pudo contener la carta del obispo Tajón a Eugenio de Toledo, un breve índice de las obras de san Gregorio, la visio Taionis y el elogio de Gregorio Magno a Isidoro de Sevilla por su De viris illustribus. ${ }^{8}$ Como se puede observar, los textos que preceden al ms. lat. 83 tienen la finalidad de contribuir a reforzar el concepto de auctoritas, frente al ms. lat. 2855 cuyas obras van dirigidas más bien a realizar una alabanza del autor del obra.

Si, como queda establecido, la forma y los contenidos de las piezas preliminares que acompañan al texto principal son muy diferentes, la divergencia se puede observar de igual modo en la parte final de los manuscritos. El colofón perdido del manuscrito mancuniano, según el explicit que recoge James, aparecería al final de la obra de forma explícita (Explicit foeliciter liber Moralium Papae Gregorii, pars ultima) acompañado de los datos de autoría (Deo gratias, Gomez diaconus, peccator, hoc opus) y de la fecha expresada de dos formas diferentes (Era DCCCC.LII.VI. kalend. Decembris, ob iussionem Domni Damiani Abbatis preaescripsi). ${ }^{9}$ En el caso del manuscrito parisino el final del texto se produce de manera abrupta, hasta el punto de que un copista posterior en escritura gótica textual comienza a escribir lo que falta (Quia enim) para enseguida abandonar la copia y colocar como nota al margen derecho: hic desunt octo folia, que seguramente faltaban en el original y por eso Gómez nos los copió, ya que aunque la obra de Ildefonso de Toledo está incompleta, el manuscrito no lo está. ${ }^{10}$ Por ello, se considera como colofón de la obra lo que en realidad es el final del prólogo que escribiera Gómez. ${ }^{11} \mathrm{Si}$ comparamos los datos que se hacen constar en los colofones de ambos códices, se pueden establecer diferencias que se refieren a la concisión en el modo de reseñar los datos, así como a la forma de expresar la data cronológica.

Según cómo aparecen dispuestos los datos y qué datos ofrece cada copista, denotan dos formas diferentes de abordar la información dada al final del texto. Así, la primera diferencia consiste en la concisión, mientras Gómez de Cardeña ${ }^{12}$ ofrece de forma escueta y sistemática los datos básicos del códice que hacen referencia al copista y al autor de la obra, el copista Gómez de Albelda parece darle más importancia a los datos referentes al dueño del libro (el obispo Gotescalco) que a los datos del códice en sí mismo (Transtulit enim hunc libellum sanctissimus

8 De este códice no se han conservado sus primeros siete cuadernos y seis folios del octavo, por lo que no se puede comprobar lo que parece aceptado por la crítica. Véase, por ejemplo, Suárez González 2002: 77-92, para el códice conservado en Manchester, 80.

9 James y Taylor 1980: I, 150-152.

10 Esta es la interpretación que realiza Díaz y Díaz 2002: 51, n. 10 de las últimas palabras del manuscrito. A pesar de que la palabra desunt aparece abreviada, nada hace pensar en otras posibilidades.

11 Como explica en su excelente estudio filológico Yarza Urquiola (2016: 217) el prólogo, que precede la obra, lo realizó después de haber copiado el resto del códice según sus propias palabras: «Libenter conscipsi libellum a beato Ildefonso Toletanae sedis episcopo dudum Iuculentissime editum (he copiado con sumo gusto el libro elengantísimamente escrito por Ildefonso)».

12 En adelante identificaremos a los copistas con las siglas de su nombre y el lugar custodio del manuscrito, así G(ómez) M(anchester) y G(ómez) P(arís).
Gotiscalcdus episcopus ex Spania ad Aquitaniam). Por lo que se refiere a la forma de expresar la data cronológica, GM considera necesario explicitar el día del mes y el año, corroborando dichos datos con el nombre del abad Damián, pero GP prefiere informarnos de la estación del año en que se encontraba, el año en curso sin el día y la constatación de dicha fecha consiste en una aproximación cronológica de tipo histórico (tempore hiemis, diebus certis ianuarii uidelicet mensis, currente feliciter era DCCCCLXXXVIIII, regnante Domino nostro lesuchristo, [...] Ipsis igitur diebus obiit Galleciensis rex Ranimirus). Además, GM aprovecha este momento para dejar constancia de sus datos personales, acompañados de breves alusiones religiosas, frente a GP que decide incluir dichos datos en el prólogo, seguidos de una fórmula religiosa mucho más explícita (lesuchristo, qui cum Deo Patre et Sancto Spiritu unus Deus gloriator in saecula saeculorum. Amen).

En ambos colofones solo hay dos coincidencias. La primera que consiste en utilizar el estilo habitual de datación en esos momentos, la era hispánica; y la segunda, expresar la alegría por la finalización de su trabajo con la palabra feliciter, aunque en el caso del copista que trabaja en Cardeña va referido al final de su laborioso trabajo frente al escriba de Albelda que la utiliza como fórmula que acompaña a la fecha.

Por último, como queda explícito en el cuadro anterior, también se establece una clara diferencia de estilo al elegir el modo de marcar los quaterni que conforman los códices. Si en el manuscrito conservado en Manchester nos encontramos todos los cuadernos numerados en romanos en el intercolumnio, incluso en una ocasión acompañando el numeral de la sigla $Q$., en el manuscrito parisino la forma de separar y marcar el cambio de cuaderno consiste en un simple reclamo situado en la parte derecha del margen inferior, que se corresponde de modo muy breve con el inicio del folio siguiente, incluso con un error en el último donde falta, y así es añadido por un copista posterior, el adverbio non. ${ }^{13}$

\section{GómIz, GómEZ, GOMESANIS, GOMES, GOMESANI}

Al analizar el problema que surge a causa de la posible homonimia entre ambos copistas, es necesario cotejar las diferentes formas en que aparece escrito el nombre: Gómez. Aunque en todas las ocasiones se trata de testimonios autógrafos, durante el siglo $X$ se producen todavía importantes alternancias gráficas en el uso de los antropónimos.

Para situar este problema en las circunstancias socioculturales en que se produce, basta con mencionar la reflexión que realiza María Rosa Fort Cañellas, quien al analizar en su estudio la onomástica aragonesa tomando como base la colección diplomática de Obarra explica:

Pero el empobrecimiento del léxico onomástico conduce a casos de homonimia, produciéndose una cierta confusión al menos en los nombres más usados; de ahí que aparezca el doble nombre. Y para que nazca la doble denominación o para que, de una manera

13 También se recoge este error en el estudio del códice que realiza Díaz y Díaz 2002: 53, n. 18. 
general, se haya sentido la necesidad de recurrir a un nuevo modo de llamar a las personas, no era necesario llegar al caos, a que cada nombre hubiese sido llevado por varios sujetos en la misma localidad o en la misma familia; basta sólo con que dos individuos en un mismo lugar tuviesen idéntico nombre para poder explicar fácilmente la expansión de la doble denominación.

Y así se generaliza a partir del siglo XI la costumbre de añadir al nombre de pila el del padre o de la madre. ${ }^{14}$

Como se puede observar a través del testimonio anterior se trata de un problema habitual en el siglo $X$ que durante la siguiente centuria, comprobado el alcance social que tiene dicho problema, será cuando se le dote de una solución lingüística. Nos encontramos, por tanto, ante un fenómeno generalizado que se causa por una evidente escasez de este campo léxico.

Ahora bien, cuando trasladamos esta situación a nuestros copistas, quizás en parte conscientes del problema que supone en esos momentos la homonimia, nos encontramos escrito su nombre explícitamente en formas diferentes. Dentro del amplio elenco de posibilidades entre las que se pueden encontrar los antropónimos asimilados a la forma actual Gómez nuestros copistas eligen dos diferentes cada uno de ellos. ${ }^{15} \mathrm{Si}$ el copista que escribe en el año 914 en la zona castellana tiende a formas más evolucionadas del nombre y así se autodenomina "Gomiz» (peccatoris) en el folio $80 \mathrm{v}$ y "Gome[z: tachado] \sanis/» (peccatoris) en el folio 142 r, el copista que realiza su obra en el año 950-51 en Albelda se inclina más por las formas declinadas latinas al elegir la forma «Gomes» (indignus presbiterii) en el folio 69v y (misérrimo) "Gomesani» en el folio 71r. Como se puede observar, el copista cronológicamente anterior y que trabaja en Burgos escribe su nombre en un latín romanceado, mientras que el escriba que trabaja en Albelda bastantes años después, opta por las formas clásicas latinas de escribir su nombre propio.

En el excelente trabajo de Yarza Urquiola que estudia la tradición textual del prólogo que escribiera Gómez a la obra de san Ildefonso en el ms. lat 2855, explica que:

El autor del Prólogo declina su propio nombre por la tercera, es decir, Gomes, Gomesanis, cuyo dativo es Gomesani [...] El genitivo Gomesanis, que hemos incluido por nuestra parte en el título del Prólogo, está atestiguado, como nos señala B. A. Shailor en el siguiente códice copiado en Cardeña en el año 914: J. Ryland's Library Lat. 83, que recoge la obra Moralia in lob de Gregorio Magno. El amanuense principal se Ilama también Gómez y en el f. $142 r$ se lee [...] peccatoris Gomesanis, aunque en el fol. 80v se encuentra esta nota: Gomez peccatoris memento, fruto de las validaciones en la flexión de los nombres propios. ${ }^{16}$

Hasta tal punto se vacila en esos momentos que el propio copista en el fol. $142 r$ utiliza la forma «Gomez» y tacha la $-z$ en favor de la forma "Gomesanis» colocando el sufijo

14 Fort Cañella 1992: II, 969-980, para la cita 970.

15 Las formas Gomecius, Gomicio, Gomessanus, Gomesano, Gomiciz, y Gomici, amén de las reseñadas más adelante en el trabajo, aparecen recogidas en diferentes trabajos lingüísticos que abordan la antroponimia que se sitúa en esta lugar y cronología, así Ramos Remedio 2010: 499-566; Cierbide 1996: 119-133; Martínez Sopeña 1994: 189198; y García de Cortázar 1991: 175-191.

16 Yarza Urquiola 2016: 219 encima de la caja de renglón de forma interlineada. Ahora bien, lo que escribe también él mismo en el folio 80v no es "Gomez» como aparece en la fuente bibliográfica de la cita anterior, si no "Gomiz», como se puede ver claramente en la siguiente figura:

FIGURA 1

CC Manchester, John Rylands Library

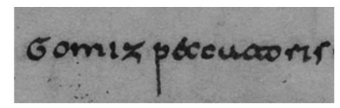

ms. lat. 83 f. 80

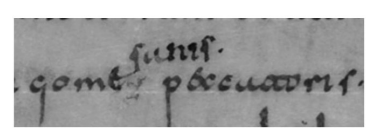

ms. lat. 83 f. 142
FIGURA 2

C Paris, Bibliothèque Nationale de France

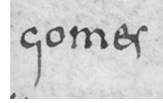

ms. lat. 2855 f. $69 v$

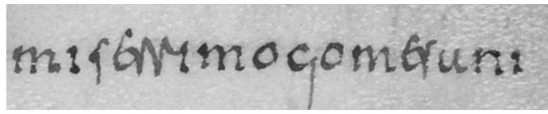

ms. lat. 2855 f. $71 \mathrm{r}$

Si además tenemos en cuenta que, como apunta la crítica filológica, el sufijo patronímico -ez da lugar al primer apeIlido peninsular y que, aunque formado en el siglo VIII, es en el siglo $X$ cuando se generaliza en el noroeste de España y en los reinos pirenaicos, parece bastante difícil poder extraer algún dato más de la forma exacta en que deciden referirse a sí mismos ambos copistas; un siglo más tarde dichas formas ya se habrían convertido en un simple apellido. ${ }^{17}$

Sí creo que son más reseñables las fórmulas calificativas con las que cada copista acompaña su nombre. Si bien todas ellas hacen referencia a la humildad necesaria por tratarse de hombres de Iglesia, el copista de san Pedro de Cardeña elige en ambas ocasiones el mismo calificativo, peccatoris, referido a una condición de tipo espiritual, frente al escriba de san Martín de Albelda que con sus diferentes formas, indignus presbiterii y misérrimo, hace más bien referencia a su condición dentro del escalafón eclesiástico.

Como se puede observar, en este campo no son muchas ni definitivas las diferencias que se pueden establecer entre ambos autores. Sin embargo, como se ha mencionado en el apartado anterior, nos encontramos claramente ante diferentes usos de escriba, lo que nos sitúa más cerca de la hipótesis de los dos copistas, también corroborada por otras posibilidades de análisis al estudiar la ornamentación.

\section{ORNAMENTACIÓN}

Las principales características que describen la decoración realizada en el ms. lat. 2855 aparecen recogidas por los investigadores que realizan el excelente catálogo sobre los manuscritos visigóticos iluminados en la península ibérica. ${ }^{18}$ En este estudio se analizan las iniciales miniadas $E, I, D, D$ que aparecen en los folios $69 \mathrm{v}, 71 \mathrm{v}, 73 \mathrm{v}$ y $78 \mathrm{v}$, respectivamente, descritas en los siguientes términos:

\footnotetext{
17 Dolç 1960-1967: I, 389-419.

18 Avril, Aniel, Mentre, Saulnier y Zatuska 1982: 15-16.
} 
- 1 à cadre charpenté (baguettes jaunes et remplisage d'entrelacs réservés ou teints de bistre), fol. 73v; fond parchemin;

- Les autres formées directement de "panneaux» d'entrelacs réservés ou teints de bistre (fol. 69v); fond parchemin.

- Tech[nique]: dessin à l'encre brune et è l'encre rouge; ocre très délayé ou bistre; peinture couvrante (jaune, vert somber)

$-[\ldots]$

- 1 initiale refaite sur grattage au début du chap. 3, fol. 89 Q: cadre plein. Tech: dessin à l'encre rouge et peinture jaune vif.

Petites initiales brunes, rouges ou réservées.

Según consta en la cita anterior, la principal característica que comparten todas estas letras ornamentadas, menos la última, es la decoración de lacería de factura simple, con apenas uso del color, ya que solo una de ellas se realiza con tres colores frente a la monocromía del resto, en las cuales se utiliza una única tinta para realizar el perfil de la letra y sus entrelazos que contrasta con el color propio del pergamino.

De origen carolingio, este tipo de ornato en letras iniciales aparece en el norte de la península ibérica hacia los años 930-940, por lo que los escribas castellanos y leoneses se habrían inspirado en la escuela de san Martín de Tours al copiar modelos franco-sajones. Así, este manuscrito realizado entre el año 950 y el 951 sería uno de los primeros testimonios peninsulares que contiene dicho tipo de iluminación. No obstante, no podemos olvidar que, casi al mismo tiempo, Florencio de Valeránica está utilizando de forma mucho más compleja y colorista este mismo tipo de ornamentación al copiar e iluminar sus Moralia in lob que finaliza en el año $945 .{ }^{19}$

Una vez descrita y analizada la iluminación de este códice, se puede observar que tan solo podríamos establecer un par de casos de iniciales miniadas en las que el manuscrito de París se acerque al manuscrito de Manchester. El resto de la ornamentación utilizada pertenece a otra escuela diferente muy posterior, puesto que en el año 914 toda esta influencia proveniente del norte de Europa no puede rastrearse en modo alguno.

Tampoco resulta coincidente la decoración de determinados signos abreviativos en los tituli que marcan el principio o el final de los capítulos, puesto que nos encontramos con indicios de redondez y proporcionalidad en el caso del manuscrito parisino, frente a la sencillez de las formas más abruptas del códice mancuniano.

19 De Silva y Verástegui 1993: 213-231, incide en que se trata de primer testimonio en que se puede constatar esta influencia nórdica de los realizados en el monasterio de San Martín del Albelda (218). También De Silva Verástegui 1984, donde aparecen recogidos además los datos de los principales estudios al respecto de autores como J. Guilmain y C. Nordenfalk.
Figura 3

(C) Paris, Blbliothèque Nationale de France @Manchester,
John Rylads Library. Tituli dentro de un incipit. Letra D miniada

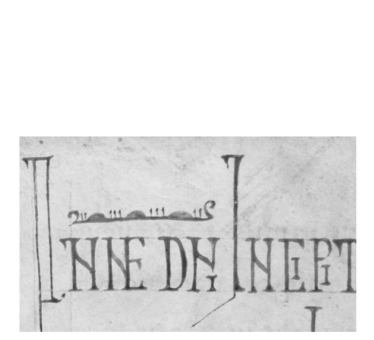

GP

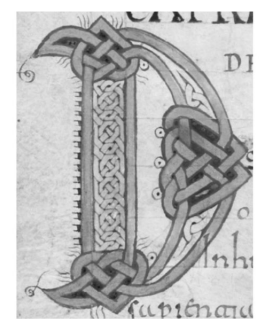

GP

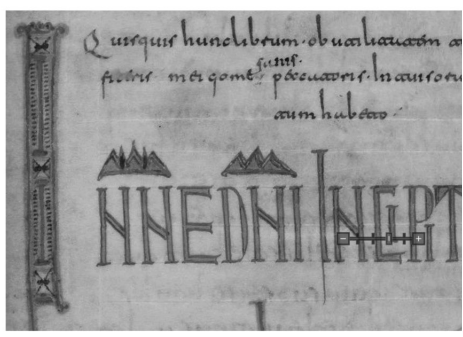

GM

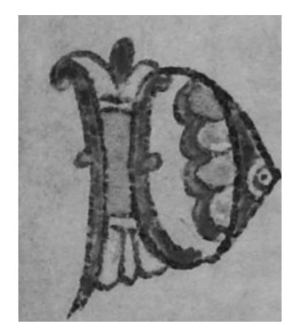

GM
A pesar de todo lo expresado anteriormente, existe alguna concomitancia, que seguramente se debe a un sustrato común, entre los manuscritos estudiados de san Ildefonso y san Gregorio. Así, la letra $Q$ miniada que aparece en el f. 89r, descrita en el catálogo citado, y un dibujo de letra $A$ que se encuentra en el f. $83 \mathrm{v}$ ambas del códice más moderno, sí son similares a las que se pueden encontrar en el más antiguo. Se trata de una letra $Q$ bicolor, amarilla y roja, con un simple adorno interior y con una línea horizontal, que completa la morfología de la mayúscula visigótica, terminada en forma de $v$ invertida con un pequeño círculo en su interior. Es cierto, que este modelo de inicial miniada se encuentra con mucha frecuencia en el manuscrito de san Gregorio, con similares formas, colores y proporciones. De igual modo que la $A$ de morfología uncial que aparece dibujada en rojo, con terminaciones similares a las de la línea horizontal de la letra anterior, también se puede encontrar, aunque con menor frecuencia, en el manuscrito de principios de siglo. Parece, por tanto, que la forma de ornamentar estas iniciales pertenece a un acervo cultural gráfico común de los dos copistas, ya que además son coincidentes con otros códices que pertenecen a este mismo entorno geográfico y cronológico.

No creo, por tanto, que estas reflexiones sobre la decoración de ambos códices sean concluyentes para filiarlos entre sí por su autoría, como tampoco creo que se pueda deducir dato alguno de sus diferencias, ya que treinta y seis años después el copista que trabaja en Albelda podría haber conocido otras modas de iluminación y, atraído por ellas, haber modernizado en este aspecto los nuevos códices que copiaba. 


\section{ANÁLISIS PALEOGRÁFICO}

Para conseguir la finalidad del presente estudio es necesario, en último término, realizar una reflexión comparativa de las escrituras de ambos códices, intentando establecer si las diferencias o las similitudes gráficas que existen entre ellos nos pueden llevar a concluir definitivamente que existieron dos manos diferentes $y$, por tanto, que existieron dos copistas distintos denominados Gómez en el siglo $\mathrm{X}$, o si por el contrario dicho análisis apuntaría hacia una misma mano en sucesivos momentos de su vida.

El primer estadio gráfico hace referencia a la morfología de las grafías aisladas que se pueden extraer de cada uno de los manuscritos. A continuación se ha elaborado un cuadro en el que se pueden observar los alfabetos de dichos copistas. La escritura que representa el ms. lat. 2855 se le ha denominado $\mathrm{GP}^{20}$ y la del ms. lat. $83, \mathrm{GM}^{21}$

FIGURA 4

(C) Paris, Bibliothèque Nationale de France CManchester, John Rylands Library. Alfabetos de letras minúsculas

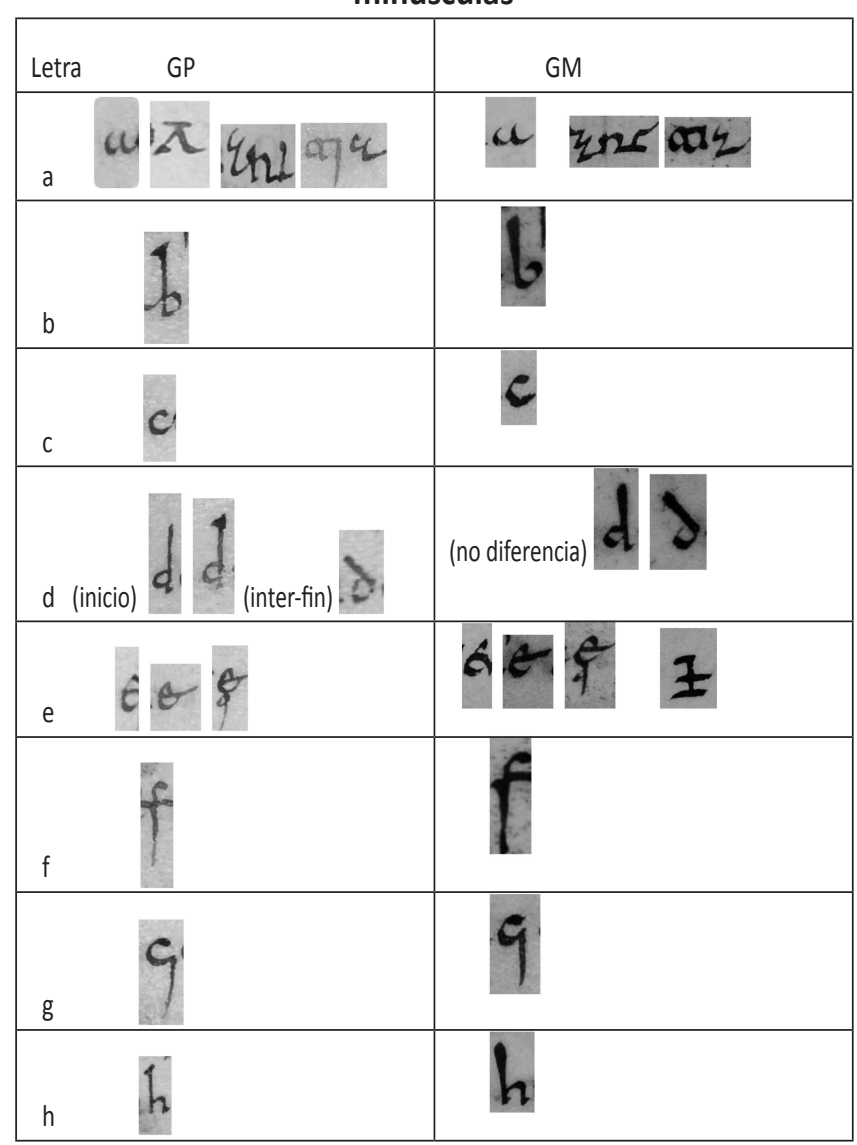

20 En la copia de la obra de san Ildefonso realizada en este códice intervienen tres manos diferentes, aquí únicamente se va a tener en cuenta la parte elaborada por Gómez (ff. 69v-159v). Se ha tenido muy en cuenta en este estudio paleográfico las aportaciones realizadas por Díaz y Díaz 2002: 55-75.

21 En el análisis gráfico del manuscrito conservado en Manchester tomo como referencia mi trabajo Cuenca Muñoz 2018, dónde se describe de forma más minuciosa y completa las posibilidades gráficas del manuscrito en general y de Gómez en particular, a partir de trabajos anteriores.

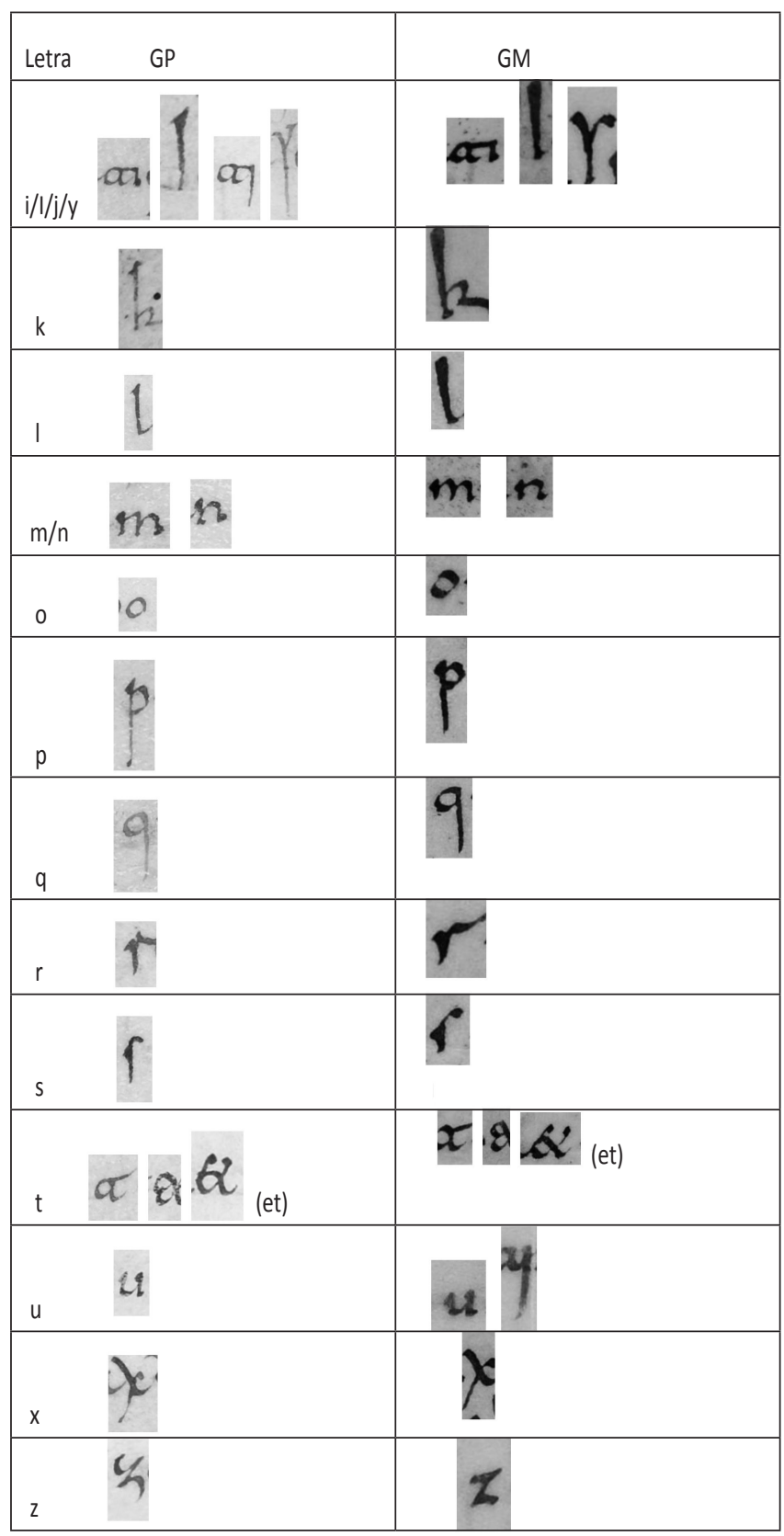

Como se puede observar existe una característica gráfica que, de manera general, atañe a las letras con astiles y que se refiere a la finalización de los mismos. El copista que trabajó en el año 950, GP, remata la parte superior de las letras $b, d, h, i$ (alta), $k$ y I con una especie de pequeño triángulo inclinado hacia la izquierda o con forma de bisel. Este mismo rasgo se puede encontrar con una similar morfología y de forma aislada en algún caído, como ocurre en el caso de la letra $p .^{22} \mathrm{Si}$ comparamos esta misma característica de las letras con astil con GM, se comprueba al cotejar las mismas formas gráficas una finalización en forma de garrote con una parte superior más ancha que la inferior y con una terminación en la

22 Según explica Lowe (1972: 53), esta característica es notable porque aparece en manuscritos de periodos anteriores, lo que lleva a pensar que se trata más de un gusto por una estética gráfica, que una imposición meramente cronológica. 
mayor parte de los casos en forma redondeada. Al observar esos mismos astiles, también queda marcado el ángulo de inclinación de cada una de las escrituras, así mientras que GM escribe con una leve desviación hacia el lado izquierdo, GP realiza las formas alfabéticas con una verticalidad casi absoluta. ${ }^{23}$

Estas observaciones gráficas, que en el fondo hacen referencia al concepto de pesadez en la escritura en un caso, y de ligereza en el otro, se unen a otra serie de cuestiones que se pueden relacionar con usos de los escribas directamente relacionados con la evolución de las formas gráficas visigóticas; por ello, creo que no se debe valorar en ningún sentido la diferencia en el uso de las formas ti/ tj como reflejo gráfico de la asibilación lingüística que tiene muy en cuenta GP, pero que desconoce o no quiere utilizar GM.

Por lo que se refiere al uso de algunas de las morfologías concretas, resulta interesante la realización de una morfología de letra $a$ en la forma cursiva en similares situaciones textuales (-ant y -tia), pero ejecutadas con un trazo absolutamente anguloso en GM que GP convierte en formas de nuevo redondeadas, además de la diferencia de la morfología de la letra $t$ en el primer caso mencionado. Diferencias en cuanto a los modelos de las letras que prefiere cada uno de los copistas también se puede establecer en la $i$ alta con la parte superior en forma de horquilla, o en las formas más rústicas y primitivas de las letras $k, l, r$ o $s$ que traza GM, frente a las mismas formas estilizadas y elegantes realizadas por GP. A ello se une una morfología muy diferente en el caso de la letra $z$, como puede verse en el cuadro anterior, apuntando de nuevo hacia el esquematismo, y un trazado básico en el caso del códice más antiguo, sustituido por una forma más elaborada en el manuscrito de la mitad el siglo $X .^{24}$

Otro elemento que resulta diferenciador es el uso de las dos morfologías posibles de la letra $d$. El copista del manuscrito parisino utiliza la forma recta solo para el inicio de palabra, dejando la forma uncial para interior o final. EI autor del códice de Manchester elige una u otra morfología sin ninguna pauta, pudiendo encontrar cada una de ellas en cualquier parte de la palabra.

Del mismo modo, el estudio de los nexos y las palabras abreviadas nos conducen a dos formas diferentes de abordar los mismos fenómenos gráficos.

23 Díaz y Díaz 2002: 56, establece «una leve, pero franca, tendencia levógira» en el copista albendense, pero como él mismo también observa "el aire general de la escritura es de ser casi recta», por lo que se ha preferido no tener en cuenta dicha leve tendencia para explicar de forma más evidente la diferencia en este aspecto entre ambos copistas.

24 Resulta curioso comprobar cómo los finales de las letras $h, m$ y $n$ son similares en los dos manuscritos a pesar de haber transcurrido treinta y seis años. Quizás ésta no sea una característica gráfica muy útil en la datación de la posibles etapas cronológicas del desarrollo dela escritura visigótica.
FIGURA 5

(C) Paris, Blbliothèque Nationale de France CManchester, John Rylads Library. Nexos, signos abreviativos y palabras abreviadas

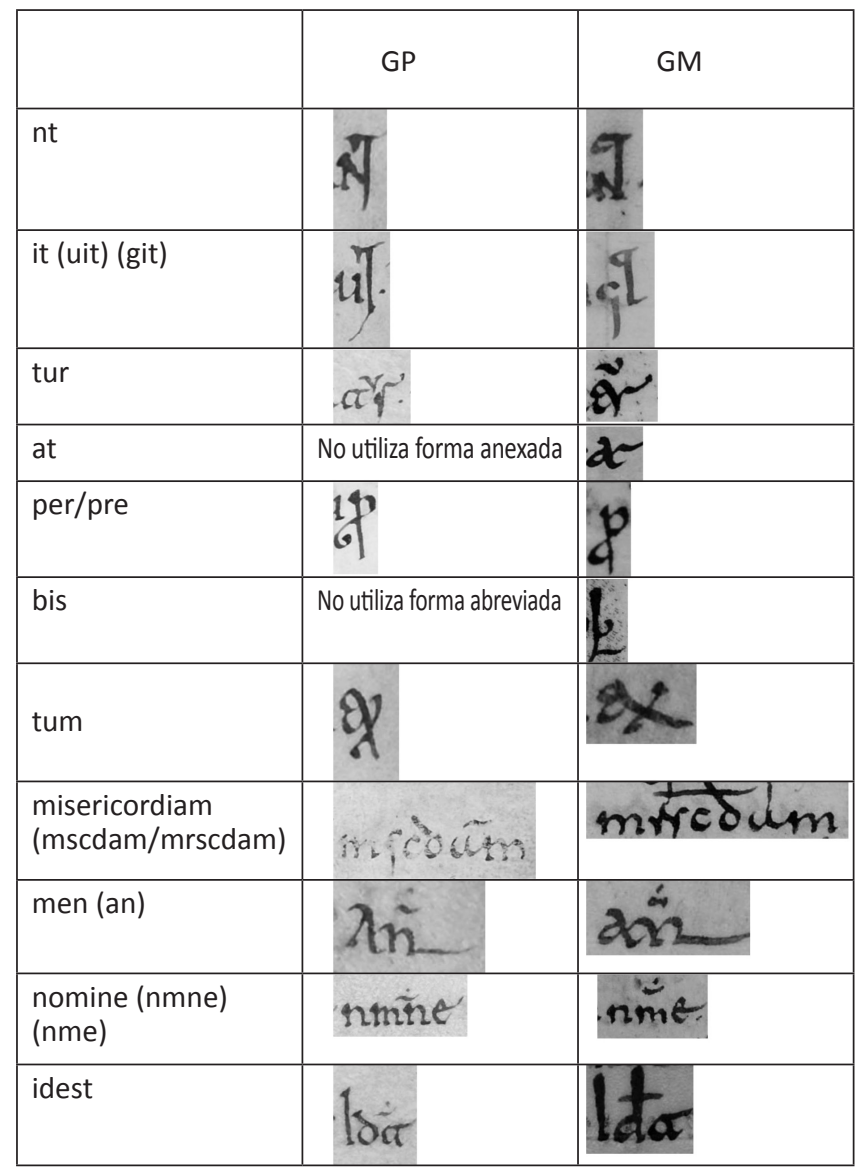

De las formas anexadas que utilizan ambos copistas, se ha escogido una representación para intentar establecer las diferencias. Se trata de los grupos gráficos en que se produce un nexo que incluye una letra dentro de otra, así en la figura anterior - nt o -it, optan por tendencias contrarias a las que describen la generalidad de sus escrituras, GP realiza trazos angulosos en la parte superior, frente a las formas redondeadas de GM. También realizan finalizaciones diferentes en el trazado de las letras, alargándolo por debajo de la caja de renglón una mano, y buscando la siguiente letra dentro de ella el otro copista.

Dos cuestiones gráficas específicas que apuntan en direcciones divergentes son las formas anexadas, es este caso mediante un trazo compartido, de las sílabas -tur y -at. El primer caso es representativo de qué ocurre cuando ambos copistas eligen la misma forma de escribir una sílaba pero, dentro del escaso margen que permite la escritura visigótica, representan el componente alfabético con diferentes morfologías. Así, donde GP elige una $t$ uncial, la letra $u$ sobrepuesta con forma aguda e igualada en sus dos partes y una letra $r$ con su típico ductus de letra aislada, GM realizó un $t$ de beta invertida haciendo nexo con la letra $r$ y una letra $u$ situada encima de la caja de renglón redondeada con la parte izquierda más alta que la derecha. 
También resulta significativo cómo determinadas formas gráficas el copista de Cardeña las realiza frecuentemente anexadas, en el caso de las letras -at, uso gráfico que no se puede encontrar en el copista de Albelda, ya que siempre las realiza como letras aisladas.

En la misma dirección apunta la utilización de algunos signos con carácter abreviativo. Cuando GP realiza el trazo gráfico que acompaña al caído de la letra $p$ para abreviar la sílaba dibuja una forma casi en espiral que resalta la elegancia pausada de su escritura, del mismo modo que la abreviación de la sílaba -tum que consiste en realizar una $t$ de beta invertida con el final continuado para cruzarlo con el signo general, se alarga por debajo de la caja de renglón produciendo una forma compacta. Frente a ese gusto por unos signos gráficos estilizados, GM, con una escritura mucho más básica y pesada en su trazado, corta el caído de la letra $p$ con una simple vuelta y alarga extremadamente hacia la derecha el final de la letra $t$ de beta invertida, en el otro ejemplo, para tener un espacio escriptorio más amplio donde aplicarle el trazo necesario que abrevia la sílaba.

Dentro del campo de las abreviaturas en los escribas altomedievales puede resultar interesante analizar no solo las formas que se utilizan, sino también las que sabiendo que podrían formar parte de su universo gráfico por aparecer en códices coetáneos, no se utilizan, aunque no podamos aclarar con seguridad cuál es la razón, ya que podría tratarse de un motivo involuntario, como el desconocimiento, o totalmente voluntario, como el desuso. El ejemplo más claro es la forma abreviada de la sílaba -bis, así frente a GM que abrevia la falta de componente semántico colocando como signo simbólico una cedilla debajo de la letra $b$, GP no utiliza nunca la forma abreviada de dicha sílaba, escribiéndola siempre de forma explícita. Dentro de este mismo campo, además de las diferencias establecidas en la utilización de determinados signos abreviativos, existen también discrepancias en la parte del componente semántico conservado de algunas palabras abreviadas. Como se puede ver en el cuadro anterior, es el caso de las palabras misericordiam (mscdam: GP; mrscdam: GM, con metátesis incluida) y nomine (nme: GM; nmne: GP). Incluso optan por morfologías diferentes de la letra $a$ - en el caso de la forma abreviada de la palabra amen, repetida con frecuencia en ambos textos, o en la forma preferida de la letra $d$ que, en el caso del manuscrito más antiguo, conlleva un cambio morfológico del signo abreviativo que pone de relieve los aspectos más primitivos de dicha escritura.

Por último, en la siguiente imagen se reproducen dos casuísticas diferentes. La primera hace referencia a cómo escribe cada uno de los copistas la misma palabra, no de forma aislada sino repetida en cada uno de sus textos. La otra hace referencia a la escritura de su nombre propio en cada códice. A pesar de que este tema lo hemos abordado desde el punto de vista lingüístico, resulta también pertinente hacerlo desde el ámbito puramente paleográfico, situando ahora el signo gráfico con respecto a los parámetros que marcan el material escriptorio.
FIGURA 6

(C) Paris, Blbliothèque Nationale de France (CManchester, John Rylads Library. Palabras características

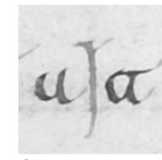

GP

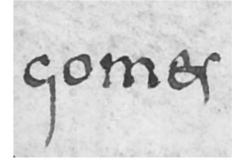

GP

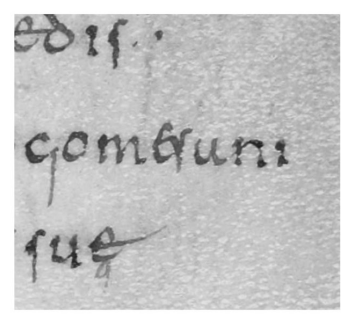

GP

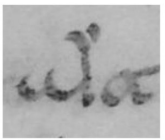

GM

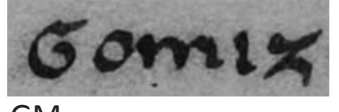

GM

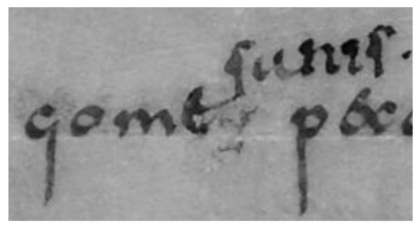

GM
Como se trata de textos que por su alto contenido en citas utilizan con frecuencia la forma verbal latina ait la comparación desde el punto de vista gráfico de esta palabra es probable que pudiera aportar algún dato. Pues bien, a pesar de su brevedad encontramos diferencias en la abertura de la letra $a$, en la parte superior de la $i$ alta con dos opciones diferentes al realizar la forma de la horquilla, y en el ductus de la letra $t$ uncial que varía posiblemente no en el número de trazos pero sí en su dirección. El segundo grupo de palabras características lo conforman las ocasiones en que cada escriba realiza su nombre contextualizado dentro del folio manuscrito. El uso de una letra mayúscula en el inicio solo lo utiliza en un caso el copista mancuniano, quien además elige la forma Gómiz en un caso y Gómez en el otro, latinizando a posteriori su nombre con un añadido encima de la caja de renglón. Es curioso observar que prefiere la forma acabada en $-z$ para su patronímico. El escriba parisino, por el contrario, se refiere a sí mismo en el colofón como Gomes en las dos ocasiones, utilizando en el segundo caso el margen derecho para añadir las letras que designan una forma más arcaica. En esto sí coinciden los dos copistas, ya que ambos utilizan la forma más antigua de su nombre como recurso escrito frente a la oralidad; de ahí que, por un olvido, se vean obligados a añadir las letras necesarias para convertirlo en un nombre declinado.

Tras la descripción y el análisis de las diferencias existentes entre los trabajos gráficos realizados en los mss. lats. 2855 y 83 , la conclusión no puede ser otra que constatar, como bien supuso M. C. Díaz y Díaz, que no se trata de dos escrituras visigóticas redondas cuyos cambios gráficos se puedan justificar por una simple evolución del trazado de la escritura, sino que está atestiguada la existencia de dos copistas diferentes que comparten un mismo nombre propio. Este es el único punto en común que existe entre ellos, puesto que reconocer dos manos no permite seguir 
estableciendo, al menos por este motivo, una vinculación forzosa entre los monasterios de Cardeña y Albelda.

En situaciones como la que nos ocupa, en las cuales al investigador le gustaría poder disponer de testimonios incontestables que corroboren su propuesta, debo añadir que existe un retrato del Gómez albeldense pero no se conoce ninguno del Gómez burgalés con el que poder establecer una comparación desde el punto de vista físico. ${ }^{25}$ Quizás se deba a la modernidad del códice parisino y a que se realizó en el monasterio de san Martín del Albelda, de dónde también se conservan otros retratos del personal que trabajaba en su escriptorio en el siglo $X .^{26}$

A modo de epílogo, es inevitable mencionar que cuando en el ámbito paleográfico se estudia la Alta Edad Media, en cuanto se cree tener alguna certeza comienza a surgir una nueva serie de preguntas. ${ }^{27}$ Si como sabemos por un documento, en el que consta la fecha del año 950, realizado en Albelda era Vigila el scriptor oficial ¿ por qué copia Gómez y no Vigila el De virginitate ese mismo año, encargado, además, por un obispo?; ¿por qué no copia Gómez el documento?; ¿cuántos copistas llegan a trabajar juntos durante esa época? En definitiva, ¿cuál fue el papel que jugó Gómez trabajando junto a un copista tan importante como Vigila?

\section{BibLIOGRAFÍA}

Avril, F., Aniel, J. P., Mentre, M., Saulnier, A. y Zatuska, Y. 1982. Manuscrits enluminés de la Péninsule Ibèrique. Paris: Bibliothèque Nationale.

Blanco García, V. (ed.). 1971. San Ildefonso de Toledo. La virginidad perpetua de Santa María. Madrid: Biblioteca de Autores Cristianos.

Cantera Orive, J. 1948-1949. "Un ilustre peregrino francés en Albelda». Berceo 9 y 11: 299-304 y 427-442.

Cierbide, R. 1996. Leyre, onomástica del Becerro antiguo: Consideraciones. Navarra: Gobierno de Navarra.

Cuenca Muñoz, P. 2018. «Un códice visigótico de los Moralia de san Gregorio». Espacio, Tiempo y Forma. Serie Medieval 31: 167-195.

De Silva y Verástegui, S. 1984. Iconografía del siglo $X$ en reino de Pamplona-Nájera. Pamplona: Institución Príncipe de Viana, Instituto de Estudios Riojanos.

De Silva y Verástegui, S. 1993 [1992]. "Los monasterios Riojanos y el arte de la miniatura en el Alto medievo», en J. I. de la Iglesia Duarte (coord.), III Semana de estudios medievales: 213-231. Nájera: Instituto de Estudios Riojanos.

Díaz y Díaz, M. C. 1979. Libros y librerías en la Rioja Altomedieval. Logroño: Ochoa.

25 En uno de los manuscritos que conserva una copia del De virginitate Sanctae Mariae conservado en la Biblioteca Palatina de Parma con la signatura ms. lat. 1650, f. 102 v realizado hacia el año 1100 aparece un retrato del escriba, Gómez, entregándole la copia del códice al obispo Gotescalco, que no sabemos si pertenece a una tradición anterior, o es fruto de la imaginación del iluminador sin interés en realizar un dibujo fidedigno. Es interesante que nuestro escriba se representa como un hombre mayor o al menos con escasa cabellera pero abundante bello facial y fuerte complexión física. Véase una reproducción de dicha miniatura en García de Cortázar 2004: 75.

26 Dentro del ámbito paleográfico es inevitable, si se menciona a los copistas que trabajaron en Albelda en el siglo X, citar el importante trabajo de los profesores Fernández Flórez y Herrero de la Fuente 2003 105-130.

27 Ibídem: 123, «De todas formas, no hay que perder de vista que por esos momentos residía en el monasterio de Albelda un copista prestigioso, el monje Gómez, el que copió el De viriginitate para Godescalco».
Díaz y Díaz, M. C. 2002. «El Códice Latino 2855 de la Bibliothèque Nationale de France en París», en C. García Turza (coord.), Los manuscritos visigóticos: estudio paleográfico y codicológico. I. Códices riojanos datados: 49-76. Logroño: Fundación San Millán de la Cogolla.

Dolç, M. 1960-1967. "Antroponimia latina», en M. Alvar (dir.), Enciclopedia Lingüística hispánica: I, 389-419. Madrid: CSIC.

Fernández Flórez, J. A. 1977. «Escribir, en León-Castilla, en época medieval», en M. A. García Guinea (dir.), Viajes y viajeros en la España medieval. Actas del V curso de Cultura Medieval celebrado en Aguilar de Campoo (Palencia) del 20 al 23 de septiembre de 1993: 145-175. Aguilar de Campoo-Madrid: Fundación Santa María la Real Centro de studios del Románico-Ediciones Polifemo.

Fernández Flórez, J. A. y Herrero de la Fuente, M. 2003. «Copistas y colaboradores en el monasterio de Albelda», en H. Spilling (ed.), La collaboration dans la production de l'Écrit Médiéval: 105-130. Paris: L'École de Chartes.

Fort Cañella, M. R. 1992. "Antroponimia primitiva aragonesa», en M. Ariza Viguera (coord.), Actas del II Congreso Internacional de Historia de la Lengua Española: II, 969-980. Madrid: Pabellón de España.

García de Cortázar, J. A. 1991. «Antroponimia en Navarra y Rioja en los siglos X a XV», en Estudios de Historia Medieval en homenaje a Luis Suárez Fernández: 175-191. Valladolid: Universidad de Valladolid, Servicio de Publicaciones.

García de Cortázar, J. A. 2004. "La implantación monástica en el Camino de Santiago: ¿monasterios del Camino o monasterios en el Camino?», en J. A. García de Cortázar y R. Teja (coords.), Monasterios y peregrinaciones en la España medieval: 72-99. Aguilar de Campoo: Fundación Santa María la Real, Centro de Estudios del Románico.

García Turza, F. J. 2002. «El monasterio de San Martín de Albelda: Introducción histórica», en Códice albeldense 976. Original conservado en la Biblioteca del Real Monasterio de San Lorenzo del Escorial (d.I.2): 9-27. Madrid: Patrimonio Nacional - Testimonio Compañía Editori.

James, M. R. y Taylor, F. (eds.). 1980. A descriptive catalogue of the Latin Manuscripts in the John Rylands Library at Manchester. Manchester: University Press.

Lowe, E. A. 1972. "Studia palaeographica. A contribution to the History of Early Latin Minuscule and to the Dating of Visigothic Manuscripts», en L. Bieler (ed.), Paleographical Papers I: 2-65. Oxford: Clarendon Press.

Martínez Sopeña, P. 1994. «Notas sobre la antroponimia hispánica medieval». Medievalismo: Boletín de la Sociedad española de estudios medievales 4: 189-198.

Pérez de Urbel, J. 1950. «La conquista de la Rioja y su colonización espiritual en el siglo X», en Estudios dedicados a Menéndez Pidal: 495-534. Madrid: CSIC.

Pérez de Urbel, J. 1977. «El escriba Eiximeno y los comienzos del escritorio de San Millán de la Cogolla». Boletín de la Institución Fernán González I: 71-89.

Ramos Remedio, E. 2010. "Aportaciones del análisis antroponímico del área vasco-románica (siglos IX-XII). La documentación de Santa María de Valpuesta». Revista internacional de estudios vascos 55 (2): 499-566.

Suárez González, A. 2002. "La edición riojana de los Moralia in Job en un manuscrito calagurritano del siglo XII». Berceo 142: 77-92.

Yarza Urquiola, V. 2016. «El prólogo de Gómez, presbítero de Albelda». Cuadernos de Filología Clásica. Estudios Latinos 36 (2): 213-232. 